\title{
Editorial
}

\section{Traceability of Goods by Radio Systems: Proposals, Techniques, and Applications}

\author{
Iñigo Cuiñas, ${ }^{1}$ Robert Newman, ${ }^{2}$ Mira Trebar, ${ }^{3}$ and Luca Catarinuccii ${ }^{4}$ \\ ${ }^{1}$ Department of Signal Theory and Communications, University of Vigo, Rua Maxwell, 36310 Vigo, Spain \\ ${ }^{2}$ School of Technology, University of Wolverhampton, Wolverhampton WV1 1SB, UK \\ ${ }^{3}$ Faculty of Computer and Information Science, University of Ljubljana, Tržaška cesta 25, 1000 Ljubljana, Slovenia \\ ${ }^{4}$ Department of Innovation Engineering, University of Salento, Via per Monteroni, 73100 Lecce, Italy
}

Correspondence should be addressed to Iñigo Cuiñas; inhigo@uvigo.es

Received 22 September 2013; Accepted 22 September 2013

Copyright (C) 2013 Iñigo Cuiñas et al. This is an open access article distributed under the Creative Commons Attribution License, which permits unrestricted use, distribution, and reproduction in any medium, provided the original work is properly cited.

Years ago, the simple need for goods (food, clothes, tools, and medicines) was the main concern for the majority of people. At that time, the quality of such goods was a minor issue. However, as economic growth provides ever more people with the surplus income to choose and, more importantly, improved communications technology provides information supporting that choice, traceability of these goods became a must. This traceability represents something similar to the identity card of a product: it is the information on what, where, and when anything happens along the production chain. Assuring the traceability sequence and its veracity represents a key factor in the competitiveness of many industries in the developed countries: it is a way to compete against lowcost products with less origin guarantee and processing information.

Traceability processes traditionally involved paper procedures, systematic tasks, and so were tedious and error-prone jobs, requiring manual checking each item of a product and the taking of notes about its production processes. Radio technologies could be a solution to systematize the capture of traceability data, its management, and, importantly, its sharing amongst partners in a value chain. Sensors, wireless networks (WiFi and WiMAX), radiofrequency identification (RFID), near-field communications (NFC), personal area networks (PAN), and so on are only some examples of possible radio technologies involved in improving traceability methods. A significant effort has been made in the analysis, research, and application of such technologies to traceability purposes, as the data provided to the consumer can be the main success factor for the producer. These efforts include antenna and sensor design, radio propagation studies, electronic development, and regulatory aspects, among many others.

The contributors to this special issue were invited to analyze the different aspects related to the application of radio systems on traceability tasks. We were interested in papers that theoretically or empirically characterize the use of such systems in traceability applications, propose new antenna designs, introduce applications to different productive sectors, evaluate the impact on the supervised processes, and describe or develop techniques to improve the track of the products during their processing.

Some papers in this special issue put the focus on the propagation problem in storehouses, factories, and fisheries, places at which wet and metallic objects are installed all around. All of the papers are based on actual systems, installed and tested in authentic workplaces. Some of them also provide innovative solutions as specially designed antennas, data loggers, or propagation tailor-made studies.

The work presented by I. Angulo et al. is focused on the difficulties of deploying radio-based traceability systems in environments as unfriendly as storehouses. Specific needs, as environment characteristics and time required to be handled by workers, are taken into account along this paper, exemplified in a prototype of a system for tracking pallets loaded with electronic devices. The prototype is based on UHF-RFID technologies.

The paper authored by L. Mainetti et al. presents a gapless solution to provide traceability along the complete fresh vegetables supply chain. They analyze critical aspects in 
the management of such supply chain, in particular, those related to the cultivation in greenhouses and manufacturing packaged vegetables. They proposed a solution based on combining RFID and NFC and supported by international standards as EPCglobal. The proposed system allows the end user to know the history of the purchased vegetables.

A novel antenna system with applications in location and tracking of small laboratory animals is the content of the paper written by L. Catarinucci et al. They analyze the propagation considerations, the effect of the animals (simulated by phantom mice) in the radio channel, and also the tracking algorithm, in a paper covering antenna design, prototyping, and testing.

The application of RFID technology in the fish supply chain combined with data loggers to provide continuity for traceability purposes in terms of cold chain control is the main topic of the work presented by M. Trebar et al. They provide actual tests of radio systems working in wet and cold environments, as fish farming industry presents, which are not so auspicious for electromagnetic propagation.

The paper offered by I. Expósito and I. Cuiñas is devoted to the limitations of the use of RFID (and, in fact, any other radio-based technology) for traceability at beverage factories. The presence of metallic elements and liquids at most of such factories appears to represent a problem when a radiowave has to be propagated within this kind of environments. An experimental analysis on propagation limitations is presented along the work, providing results of tests performed by a large variety of RFID antenna designs.

Iñigo Cuiñas

Robert Newman

Mira Trebar

Luca Catarinucci 

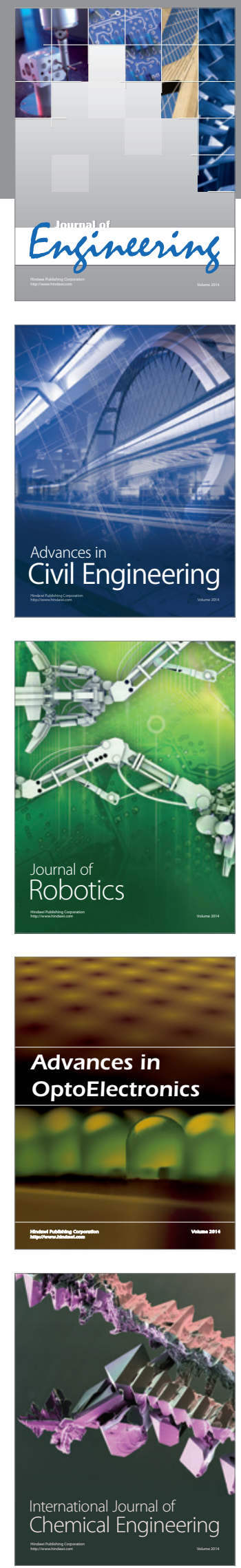

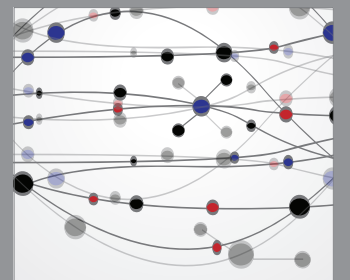

The Scientific World Journal
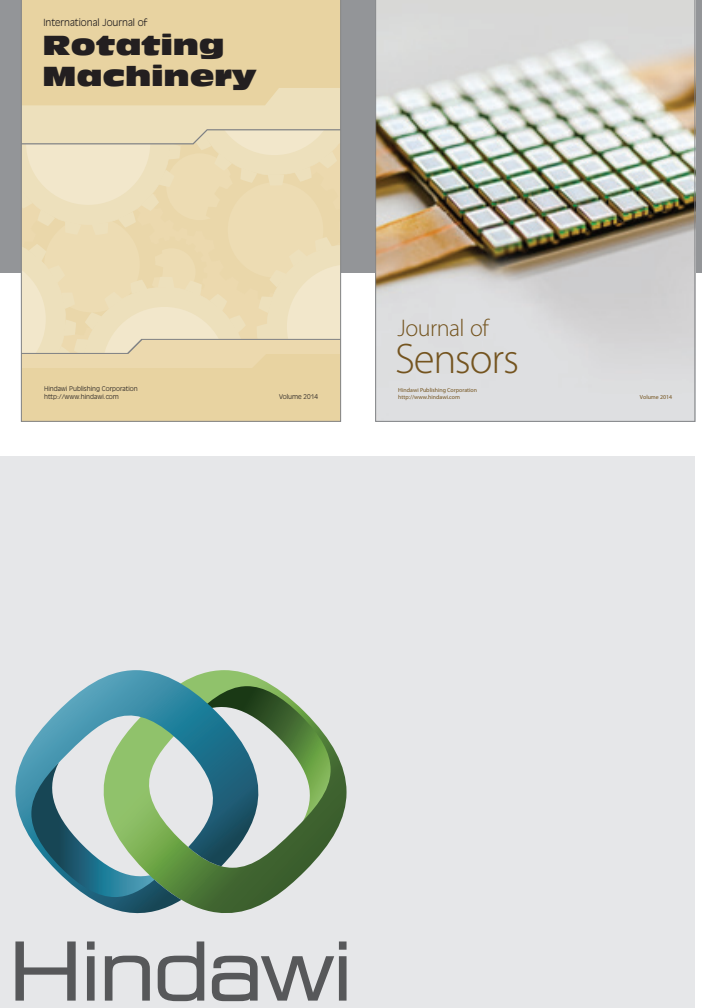

Submit your manuscripts at http://www.hindawi.com
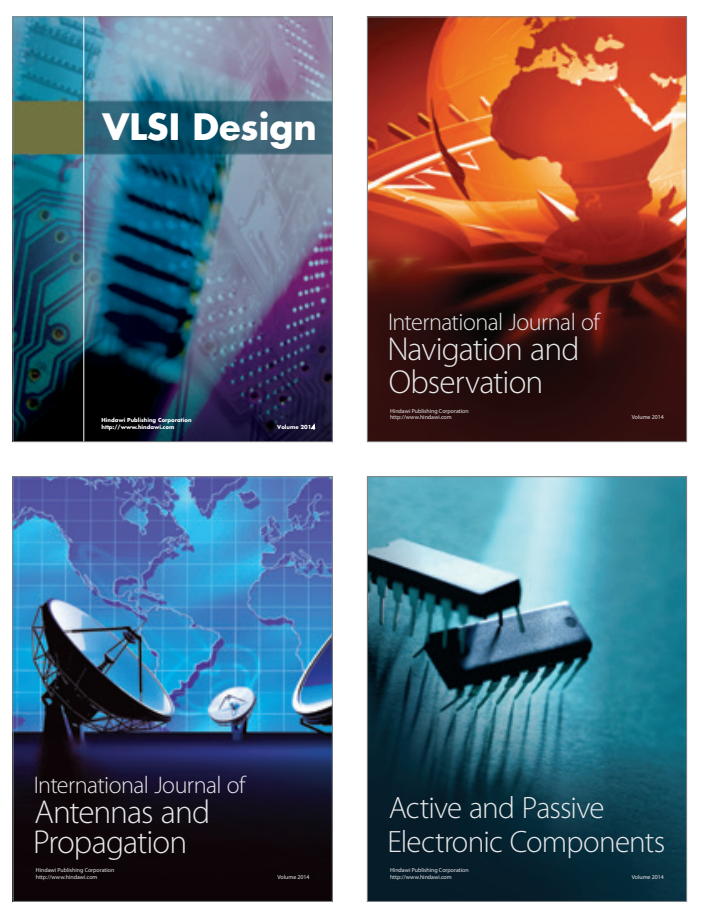
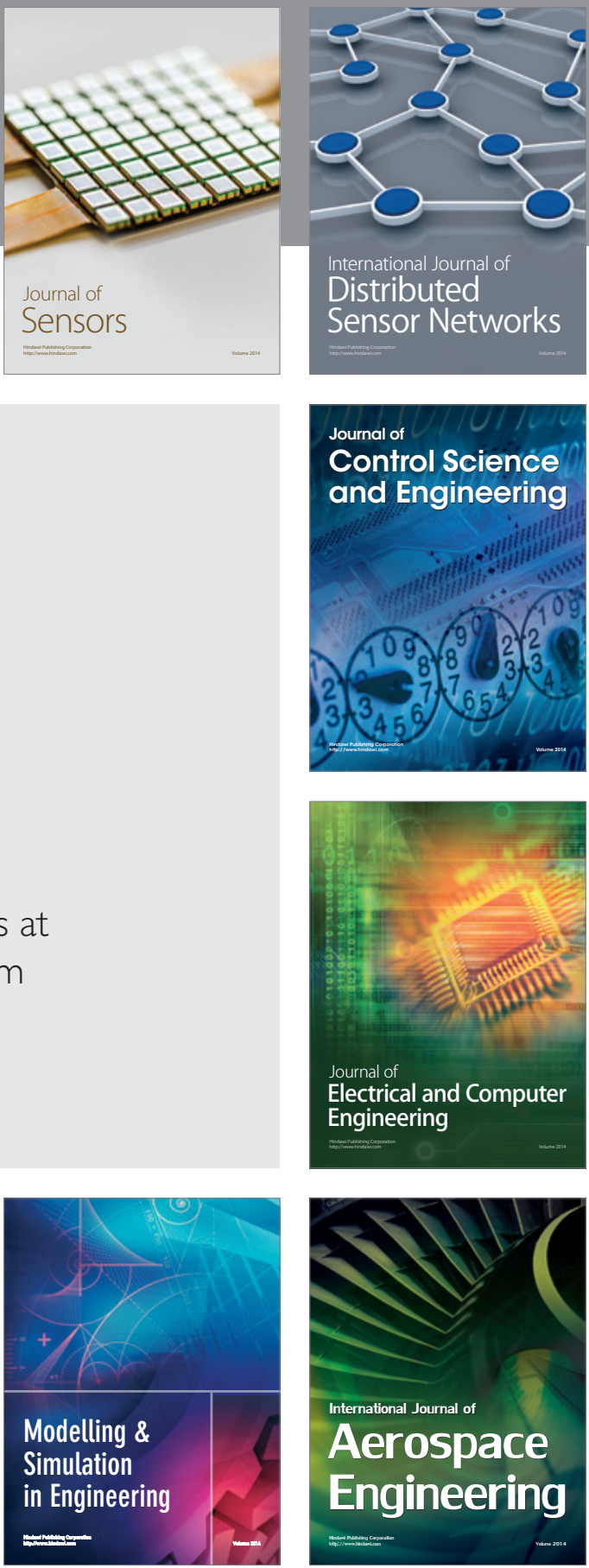

Journal of

Control Science

and Engineering
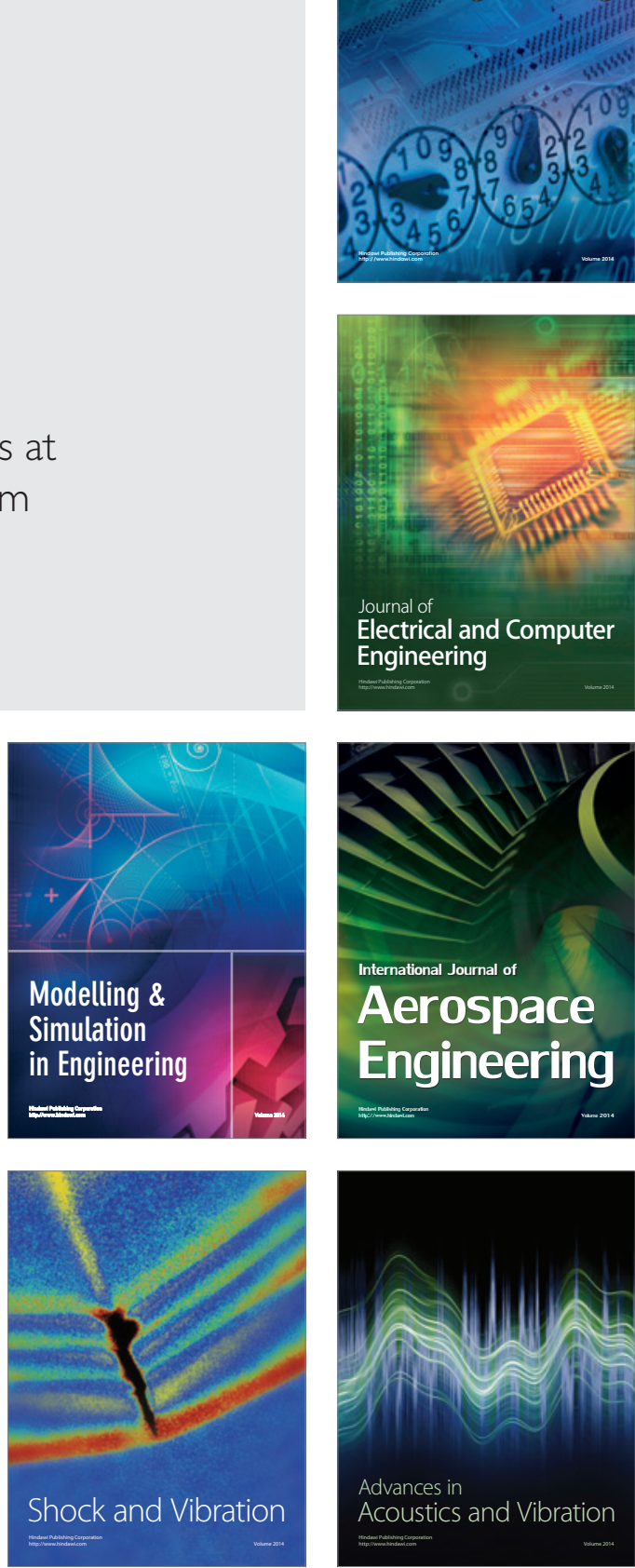